\title{
Much Ado About Nothing
}

\author{
James Tour
}

Eddy Jiménez, Clémentine Gibard, and Ramanarayanan Krishnamurthy, "Prebiotic Phosphorylation and Concomitant Oligomerization of Deoxynucleosides to Form DNA," Angewandte Chemie International Edition 60, no. 19 (2021): 10,775-83, doi:10.1002/ange.202015910.

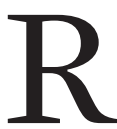

AMANARAYANAN KRISHNAMURTHY and his team at the Scripps Research Institute in San Diego recently published a paper entitled "Prebiotic Phosphorylation and Concomitant Oligomerization of Deoxynucleosides to Form DNA." As is often the case in origins of life (OOL) research, bold claims were made for the significance of the work. "This finding," Krishnamurthy remarked at the time, "is an important step toward the development of a detailed chemical model of how the first life forms originated on Earth." ${ }^{1}$ Rather than leading to any sort of chemical model, the research serves only to underscore how this particular approach is unlikely to yield any clues about how life emerged. It is further evidence, if any were needed, that eminent synthetic chemists-and scientists in general-remain clueless about life's origins. ${ }^{2}$

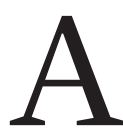

S PART OF THEIR STUDY, Krishnamurthy et al. found that when they purchased a variety of homochiral 2'-deoxyribonucleosides, which are isolated from biological sources by the depolymerization of DNA, they were able to repolymerize them into 4- to 6-unit-long oligo-2'-deoxyribonucleotides. The researchers used a paste-like condition with just a drop of water to make this happen. Rather than attempting to explain how ultra-dilute solutions of well-defined biological-looking building blocks might find each other in the earth's prebiotic oceans, the researchers instead came up with the idea of having so much of those molecules present that they form an ultrahigh concentrated paste.

There is, of course, no polymerization taking place on the bare 2'-deoxyribonucleosides. This is because the $5^{\prime}$-hydroxyl group needs to be activated before it can become a leaving group. Here, one would typically consider the prebiotically relevant phosphate moiety, but alas, no polymerization takes place with the canonical nucleotide. Instead, the researchers use a different leaving group, known as an amidophosphate, on the $5^{\prime}$-hydroxyl using diamidophosphate (DAP), which they describe as a "potential prebiotic phosphorylating agent." ${ }^{3}$ Under these conditions, the $5^{\prime}$-hydroxyl group is activated and the $3^{\prime}$-hydroxyl on a neighboring molecule can serve as the nucleophile needed to facilitate coupling.

The authors also cite preliminary data showing that the same type of oligomerization can be done using ribonucleosides-RNA's building blocks-rather than DNA's 2 -deoxyribonucleoside building blocks. This suggests that RNA-DNA (RDNA) chimeras could form, yielding interspersed units of 2'-deoxyribonucleotides with ribonucleotides. "Such mixtures," the authors remark, "can move forward for 'primordial biochemical exploitation' and for the 'simplification of the transition from chemistry to biology.",

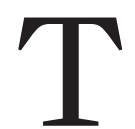

HE SAME SHORTCOMings and omissions that plague current OOL research can also be found in the paper under review. Indeed, these issues are so routinely ignored by researchers that the field appears to have become numbed to their absence from the literature. OOL researchers are prepared to assume that an ever-increasing list of obstacles were overcome on the prebiotic earth, but do not consider these hurdles as problems to be solved in their own work. The following five shortcomings in the research presented by Krishnamurthy et al., are emblematic of broader issues that need to be addressed.

First is the problem of plausibly explaining how homochiral 2'-deoxyribonucleoside was obtained in the prebiotic environment. This is not a problem for present-day researchers or laboratories-they simply buy it for use in their studies. Krishnamurthy et al. cite a number of papers where deoxyribose has been synthesized in a prebiotically relevant manner. But in all the cited papers, there is no mention of any homochiral synthesis. Even with the poorly diastereomerically controlled syntheses in those studies, the authors often identified just a blip of the desired nucleoside that was beset with many isomers and other related structures-not to mention the oligomers and untold unidentifiable products. As is often the case in OOL research, the desired products were almost never separated so that they could be carried on to the next step. The 
researchers either purchased those compounds in pure form or prepared them using modern synthetic chemistry. Such nonsensical approaches are commonplace in these studies. Consider the claim that a racemic 2'-deoxyribose was identified through high performance liquid chromatography and that no further explanation is needed. This assertion becomes the justification for both the purchase of homochiral 2'-deoxyribonucleoside and the subsequent claim that starting from this particular point in the process is prebiotically relevant. This approach is unconscionable. The starting material was obtained from biological sources or produced in a manner with no relevance for prebiotic chemistry. These issues alone should be sufficient to discredit any claims associated with subsequent steps in the process. But this is not the case. Instead, the researchers are emboldened at each stage in the process.

Second, OOL research is often beset with concentration problems. How might starting materials become available in sufficiently high yields and undiluted by the oceans, for example, so that the chemistry can occur at any usable rate? How can synthesis outpace decomposition at ultralow concentrations? Krishnamurthy et al. claim to have solved this problem by using an ultrahigh concentration paste of the $2^{\prime}$-deoxynucleosides. This is a convenient way to resolve the issue-in effect, solving the high dilution problem by assuming that there were so many homochiral 2'-deoxyribonucleosides present that they could form agglomerates too concentrated to dissolve. And where do the researchers suggest finding such a high concentration of nucleosides in the prebiotic environment? This question is left unanswered; it is a problem for the early earth, but not for the modern researcher. Assuming that, by some stroke of good fortune, high concentrations of homochiral 2 -deoxyribonucleosides were found in a paste, this would still not be enough. To obtain oligomers 4 to 6 units long the researchers then had to add DAP and 2-aminoimidazole as a base. At those levels of coupling, only 1 to 2 possible codons in length might be defined, where each codon defines a single amino acid after transcription and translation. Such a short template undermines any claim that this research represents tangible progress toward a detailed chemical model of life's origins. There is, of course, no discussion of nucleotide order in the paper, which is the basis for the requisite information code needed to eventually construct cellular components.

A third shortcoming lies in justifying the use of DAP to make the $5^{\prime}$-hydroxyl into a suitable leaving group. In this study, DAP is described as a "potential prebiotic phosphorylating agent." ${ }^{5}$ The authors have used the same reagent in several of their previous papers, where they described it in the same manner. Any attempt to discern the prebiotically relevant synthesis that was used to prepare DAP quickly turns into a wild-goose chase. Readers of the article under review are referred to an earlier Krishnamurthy paper from 2017, that, in turn, cites an article by Albert Eschenmoser published in $2000 .{ }^{6}$ Eschenmoser, for his part, cites a 1986 article by Makoto Watanabe and Shoji Sato, even though they did not use a prebiotically relevant route. ${ }^{7}$ Eschenmoser also cites a 1957 article by R. Klement, G. Biberacher, and V. Hille entitled "Beiträge zur Kenntnis der Monoamido-und der Diamidophosphorsäure (Contribution to the Knowledge of Monoamido and Diamidophosphoric Acids)." ${ }^{8}$ This paper has nothing to do with prebiotic chemistry. It seems that DAP has become so ubiquitous in OOL research that it is now simply accepted as being prebiotically relevant. Any effort to retrace the research that underlies this assumption leads nowhere.

The fourth shortcoming is in the lack of an explanation for why the polymerization reactions had only the nucleosides and no other competing nucleophiles or electrophiles present in the paste. When OOL researchers turn to amino acids, all 20 are available for use in reaction mixtures, as needed. When they need nucleobases, these are all available too. But here, the step-growth oligomerizations are devoid of any competing elements, such as exogenous alcohols, amines, and thiols. The presence of $100 \%$-pure starting materials for the phosphorylation process and subsequent oligomerizations is certainly convenient for the researchers, but it is by no means realistic. If such impurities were present in even just a few percent of these materials, the competing reactive sites would terminate the already dismal oligomerizations. 9 The notion that such materials were present with $100 \%$ purity in the prebiotic world seems highly improbable, to say the least.

Finally, in the RDNA chimeras, there is no mention made of the 2'-hydroxyl group attacking a neighboring 5 '-amidophosphate in the paste. This results in the unwanted $2^{\prime}, 5^{\prime}$-linkage, a problem that undermines the polymerization of ribonucleotides on clay. ${ }^{10}$ The $2^{\prime}, 5^{\prime}$-linked systems are far from innocuous. They disrupt templated reactions, serving to disorder the helical structure. These systems can act also as small interfering RNAs. ${ }^{11}$ The room-temperature instability of the oligoribonucleotides is another point left unaddressed by Krishnamurthy et al.-even though oligoribonucleotides are prone to rapid decomposition with the $2^{\prime}$-hydroxyl displacing the $3^{\prime}$-phosphate. ${ }^{12}$

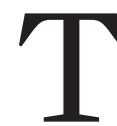

HE AUTHORS CONCLUDE their discussion by reiterating that "our work here is based on the scenario that both deoxynucleosides and ribonucleosides are available by plausible prebiotic pathways." They add:

The view that deoxynucleosides would have been available alongside ribonucleosides, though old, is not widely popular as the RNA-world hypothesis. However, it must be pointed the RNA-centric view and the RNA-only approach is limited in its validity and has been-rightfully-questioned critically. ${ }^{13}$

OOL research, as currently practiced, is-rightfullyquestioned critically. In recent decades, OOL conjectures 
have shifted from protein-based theories, to the RNA world hypothesis, and now to RDNA-based proposals. This despite the facts that amino acids do not polymerize readily due to their zwitterionic form and, without sidechain blocking, all hope of polymerization into polypeptides has been dashed ${ }^{14}-$ even if one could generate the necessary 19 homochiral amino acids, a feat that has never been achieved in a prebiotically relevant synthesis. For this reason, the protein-based proposal in which so much hope had been invested must now be added to the list of failed OOL approaches. The RNA world hypothesis, now half-a-century old, posits that the first replicators were RNA-based, and that DNA arose later as a product of RNA life forms. These notions and the RNA world hypothesis itself have proven remarkably durable, even though they fail to account for

- the prebiotic chemical difficulties involving in creating a homochiral ribonucleoside;

- the thermal instability of RNA, which decomposes rapidly, even at $0^{\circ} \mathrm{C} ;^{15}$

- the fact that even specifically designed and primed RNAs have never been shown to duplicate more than $7 \%$ of themselves, and that those segments were found to be too short to serve as new templates; ${ }^{16}$

- the difficulties involved in separating RNA-RNA duplexes, which impede further reactions;

- the role played by non-canonical $2^{\prime}, 5^{\prime}$-linkages that are routinely obtained in $20-80 \%$ yields, retard subsequent templated utility, ${ }^{17}$ and play no part in translation and transcription. ${ }^{18}$

The new RDNA world hypothesis only resolves one of these issues: the RNA-RNA sticky-duplex problem. For all these reasons, it is very difficult to accept the claims being made about the significance of this new research. As it stands, the prospects for the RDNA world hypothesis appear bleak.

James Tour is a synthetic organic chemist at Rice University.

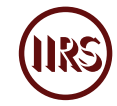

1. "Discovery Boosts Theory that Life on Earth Arose from RNA-DNA Mix," Science Daily, December 28, 2020.

2. I have written previously for Inference on the shortcomings of current origins of life research. See James Tour, "Animadversions of a Synthetic Chemist," Inference: International Review of Science 2, no. 2 (2016), doi:10.37282/991819.16.13; James Tour, "Two Experiments in Abiogenesis," Inference: International Review of Science 2, no. 3 (2016), doi:10.37282/991819.16.33; James Tour, “An Open Letter to
My Colleagues," Inference: International Review of Science 3, no. 2 (2017), doi:10.37282/991819.17.44; and James Tour, “Time Out," International Review of Science 4, no. 4 (2019), doi:10.37282/991819.19.30.

3. Eddy Jiménez, Clémentine Gibard, and Ramanarayanan Krishnamurthy, "Prebiotic Phosphorylation and Concomitant Oligomerization of Deoxynucleosides to Form DNA," Angewandte Chemie International Edition 60, no. 19 (2021): 2, doi:10.1002/ange.202015910.

4. Jiménez, Gibard, and Krishnamurthy, "Prebiotic Phosphorylation," 2.

5. Jiménez, Gibard, and Krishnamurthy, "Prebiotic Phosphorylation," 2.

6. Megha Karki et al., "Nitrogenous Derivatives of Phosphorus and the Origins of Life: Plausible Prebiotic Phosphorylating Agents in Water," Life 7, no. 3 (2017), doi:10.3390/life7030032; and Ramanarayanan Krishnamurthy, Sreenivasulu Guntha, and Albert Eschenmoser, "Regioselective $\alpha$-Phosphorylation of Aldoses in Aqueous Solution," Angewandte Chemie International Edition 39, no. 13 (2000): 2,281-85, doi:10.1002/1521-3773(20000703)39:13<2281::AID-ANIE2281> 3.0.CO;2-2.

7. Makoto Watanabe and Shoji Sato, "The Synthesis and Thermal Behaviour of Sodium Phosphorodiamidate," Journal of Materials Science 21 (1986): 2,623-27, doi:10.1007/ BF00551463.

8. R. Klement, G. Biberacher, and V. Hille, "Beiträge zur Kenntnis der Monoamido-und der Diamidophosphorsäure (Contribution to the Knowledge of Monoamido and Diamidophosphoric Acids)," Zeitschrift für anorganische und allgemeine Chemie 289, no. 1-4 (1957): 80-89, doi:10.1002/ zaac.19572890108.

9. Wikipedia, "Carothers Equation."

10. Gözen Ertem et al., "Bridging the Prebiotic and RNA Worlds: Prebiotic RNA Synthesis on Clay," Journal of Biomolecular Structure and Dynamics 17 (2012): 207-10, doi:10.1080/0739 1102.2000.10506623.

11. Thazha Prakash et al., "RNA Interference by 2 ',5'-linked Nucleic Acid Duplexes in Mammalian Cells," Bioorganic Et Medicinal Chemistry Letters 16, no. 12 (2006): 3,238-40, doi:10.1016/j.bmcl.2006.03.053.

12. "Oligonucleotide Handling \& Stability," Merck.

13. Jiménez, Gibard, and Krishnamurthy, "Prebiotic Phosphorylation," 7.

14. Matthew Powner, "Peptide Synthesis at the Origins of Life: Energy-Rich Aminonitriles By-pass Deactivated Amino Acids Completely," Nature Portfolio Chemistry Community, July 10, 2019.

15. “Oligonucleotide Handling \& Stability,” Merck.

16. Wendy Johnston etal., "RNA-Catalyzed RNA Polymerization: Accurate and General RNA-Templated Primer Extension," Science 292, no. 5,520 (2001): 1,319-25, doi:10.1126/ science.1060786; Hani Zaher and Peter Unrau, "Selection of an Improved RNA Polymerase Ribozyme with Superior Extension and Fidelity," RNA Society 13 (2007): 1,017-26, doi:10.1261/rna.548807. 
17. Gözen Ertem, "Montmorillonite, Oligonucleotides, RNA and Origin of Life," Origins of Life and Evolution of the Biosphere 34 (2004): 549-70, doi:10.1023/B:ORIG.0000043130.49790. a7.

18. Prakash et al., "RNA Interference."

DOI: $10.37282 / 991819.22 .1$

Published on January 4, 2022

https://inference-review.com/article/much-ado-about-nothing 